\title{
FÓRMULA DU PONT Y SU RENTABILIDAD, VISTA DESDE LA OPTICA ADMINISTRATIVA ${ }^{1}$
}

DU PONT EQUATION AND YOUR RETURN, FROM A POINT ADMINISTRATIVE

\section{Omar García Jiménez ${ }^{2}$}

Para citar este artículo: García, O. (2014). "Fórmula Du Pont y su rentabilidad, vista desde la óptica administrativa”. Inquietud Empresarial. Vol. XIV (2), p.p. 89-113

Fecha de recepción: 2 de septiembre de 2014

Fecha de Aceptación: 18 de noviembre de 2014

\footnotetext{
1 Artículo de Reflexión como resultado del proyecto de investigación "Análisis económico y financiero".

2 Profesor Investigador del área de Posgrado en Ingeniería Industrial, impartiendo la asignatura de análisis económico dentro del Tecnológico de Estudios Superiores del Oriente del Estado de México, México. Maestro en Ciencias en Administración y Contador Público Certificado.omar24_ga@yahoo. com.mx
} 


\section{RESUMEN}

Es importante adentrarnos dentro de la práctica del análisis financiero con un espíritu fáctico, veraz y objetivo que permita proporcionar un margen de referencia y generar medios de análisis e interpretación de la información para toma de decisiones. Para ello es necesario establecer objetivos y utilizar de manera óptima herramientas analíticas.

Ha sido un hecho destacable el dinamismo y razonamiento con el que se ha abordado de manera particular el estudio de las finanzas; cada una de las metodologías y avances que se desarrollan en nuestros tiempos denotan más la carencia de control o dominio sobre los efectos financieros y la interpretación que da un buen análisis financiero. Así el objetivo de este trabajo, es analizar las variables que intervienen en el desarrollo de la formula Du Pont, visto desde una perspectiva administrativa.

\section{PALABRAS CLAVE}

Finanzas, Formula Du Pont, Análisis, Decisiones

\section{ABSTRACT}

It is important to enter to us within the practice of the financial analysis with a factual, truthful spirit and objective who allows to provide a reference margin and to generate means of analysis and interpretation of the information for making decision. In order to make assertive decisions it is necessary to establish objective and to use of optimal way analytical tools. It has been a remarkable fact the dynamism and reasoning with which the study of the finances is had boarded of particular way; each one of the methodologies and advances that are developed in our times denote plus the deficiency of control or dominion on the financial effects and the interpretation that a good financial analysis gives.

Therefore the objective of this work, is to analyze the variables that take part in the development of formulates Du Pont, sight from an administrative perspective.

\section{KEY WORDS}

Finances, Formulate Du Pont, Analysis, Decisions 


\section{INTRODUCCIÓN}

El modelo Du Pont consiste en una formula-diagrama que permite ver fácilmente la descomposición del rendimiento de los activos y del rendimiento sobre el capital en sus diferentes etapas o elementos; además, muestra la relación entre esos elementos y las variables económicas que las afectan.

Este planteamiento no trata de descubrir el hilo negro de la tela, sino llegar a una cuestión casi invisible o imperceptible como lo es el resultado que surge tras analizar y modificar las variables financieras.

Un análisis financiero como el análisis Du Pont ${ }^{3}$, permite cotejar y desmembrar cada factor de una forma más digerible; logrando desarrollar y maximizar la meta de todo accionista: posicionarse en el mercado y maximizar las ganancias tanto de la empresa como de los accionistas. (Ochoa, 1998)

Trabajos como el que hoy nos ocupa se validan por su pertinencia al proporcionar herramientas que complementan la necesidad de capacitación empresarial y la vinculación del sistema educativo a través de la interrelación de conocimientos y experiencia, es decir conjuntar tanto los aspectos cuantitativos como cualitativos que inciden para tomar decisiones.

\section{ANTECEDENTES}

Durante la segunda época del siglo pasado se dio un periodo de gran expansión económica a un ritmo sin precedentes, producto de una coyuntura favorable propiciada por la llegada de dinero del exterior y de una demanda creciente que estimulaba la inversión (Comellas, 2004). Esto propicia la necesidad de desarrollo y principios básicos de las finanzas Administrativas. ${ }^{4}$

Los años veinte se caracterizaron por su desarrollo industrial y acelerado en los Estados Unidos de América; ocasionando avances de toda índole, desde las ciencias hasta formas de diversión en general.

El desarrollo económico generó una burbuja especulativa y a mediados de la década de 1920 el mercado de valores en los Estados Unidos experimentó una rápida expansión hasta la gran caída del precio de las acciones en octubre de 1929, desplome que contribuyó a la gran depresión diez años y más grave jamás experimentada por el mundo occidental industrializado. Aunque se originó en Estados Unidos, este fenómeno provocó la disminución drástica de la producción, el desempleo grave y la deflación aguda en casi todos los países del mundo (Enciclopedia Britanica, 2014). El modelo Du Pont es creado por Donaldson Brown ${ }^{5}$ (Catanese, 1997). Mostrando su perspicacia financiera en 1912, cuando presentó un informe de la eficiencia

3 Eleuthère Irénée (E.I.) Du Pont (1771-1834) político y economista francés que constituyó la empresa que lleva su nombre, estudió avanzadas técnicas de producción de explosivos con el famoso químico Antoine Lavoisier.

4 Finanzas Administrativas: área que se ocupa de las tareas del administrador financiero en la empresa de negocios.(Gitman,2007 citado por Mercado, 2011 )

5 Donaldson Brown (1885-1965) ingeniero con estudios de postgrado en la Universidad de Cornell, se unió a Du Pont en 1909 como vendedor de explosivos, también se desempeñó como directivo financiero de Du Pont y de General Motors Corporation (GMC) 
de la empresa ante el Comité Ejecutivo utilizando una fórmula de retorno de inversión. Tras ver su eficacia el señor John J. Raskob ${ }^{6}$ acogió a Brown y le animó a desarrollar procedimientos contables uniformes y otras fórmulas estadísticas estándar que permitieron a los gerentes de división evaluar el rendimiento en toda la empresa a pesar de la gran diversificación de la década de 1910.

En 1918 Brown ejecutó una fuerte inversión de Du Pont en General Motors Company (GMC), tomando el cargo de la oficina del tesorero, atrayendo economistas y estadísticos, una práctica excepcional en la época.

En 1921 Du Pont había adquirido una participación de control en el abanderamiento de General Motors Corporation, llevando a Brown al puesto de vicepresidente de finanzas dentro de la firma. Brown ayudó a lograr la recuperación financiera de la empresa y en 1923 desarrolló los mecanismos que permitieron a Du Pont retener la inversión de GMC perfeccionando las técnicas de contabilidad de costos que había estado desarrollando en Du Pont (Dupont, 2014).

Los principios de la rentabilidad de la inversión, el retorno de la equidad, la previsión y la presupuestación flexible, fueron posteriormente adoptados extensamente en todas las empresas de Estados Unidos.

Es en este marco que Donaldson Brown (directivo financiero de Du Pont y de General Motors Corporation), desarrolló la ecuación como una forma de medir el desempeño y la rentabilidad de la empresa a través del retorno de la inversión (Mercado, 2011) considerándose a Brown como un innovador en la materia, constructor de muchos de los controles financieros básicos de Du Pont. En esa época era el hombre más versado en los Estados Unidos sobre el desarrollo y uso de estas nuevas herramientas administrativas. Sus logros fueron ejemplo de la aplicación de procedimientos de información y estadística en la administración de empresas complejas (Chandler, 2003).

\section{Formula Du Pont}

De acuerdo con Block y Hirt (2000) nos dicen que "La meta final de la empresa es lograr una valoración máxima para sus títulos valores en el sitio del patrimonio $\mathrm{y}$, se puede acercar o no a esa meta utilizando la deuda para aumentar el retorno sobre el capital. Todas las situaciones se deben evaluar por separado". El sistema de análisis Du Pont se utiliza para examinar los estados financieros de la empresa y evaluar su condición financiera. Reúne el estado de resultados y el estado de posición financiera en dos medidas de rentabilidad: Rendimiento sobre Activos o por sus siglas en Ingles (ROA: Return on Total Assets) ${ }^{7}$ y Retorno sobre el patrimonio (ROE: Return on Common Equity), ${ }^{8}$ utilizando el Margen de Apalancamiento Financiero $(\mathrm{MAF})^{9}$ respectivamente.

6 John J. Raskob (1879-1950) secretario privado del más alto nivel ejecutivo de Du Pont. 7 ROA (Return on Assets) Retorno sobre los activos ROA = Utilidad Neta / Activo Total 8 ROE (Return on Equity) Retorno sobre el Capital Propio ROE = Utilidad Neta / Patrimonio Total

9 MAF ( Multiplicador de Apalancamiento Financiero) MAF = (Activo Total / Patrimonio Total) 
Esto nos indica a groso modo que para concebir adecuadamente la formula, se relaciona primero el margen de utilidad neta, que mide la rentabilidad de las ventas de la empresa, con su rotación de activos totales, que indica la eficiencia con la que la empresa ha utilizados sus activos para generar ventas, dándonos como resultado el rendimiento sobre los activos totales (ROA).

Figura 1. Representación gráfica de la fórmula Du Pont

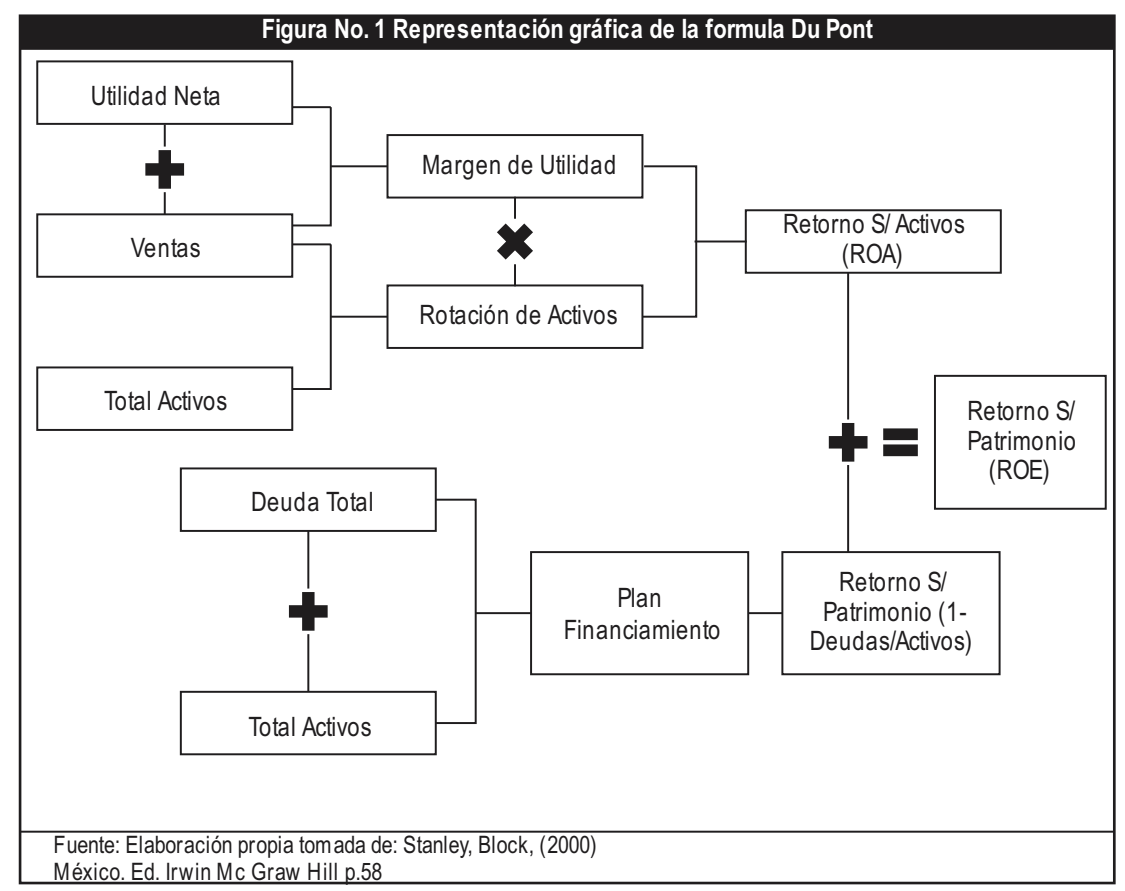

Fuente: Elaboración propia

Otra forma de poder representar gráficamente la formula Du Pont es a través del Margen de Apalancamiento Financiero, el cual consiste en dividir los activos totales entre el capital contable, el resultado así obtenido se multiplica por el ROA. Para ello transformaremos el retorno sobre el patrimonio de la siguiente manera:

; Esto equivale a la siguiente ecuación;

Patrimonio $=$; por lo tanto al dividir el ROA entre el retorno sobre la inversión tendríamos:

;

Esto equivale a:

;

Por lo tanto al eliminar el total de los activos tendríamos un término tanto en el numerador como en el denominador para quedar como sigue: 


\section{ROE}

Gitman (2007) plantea que: "el segundo paso del sistema Du Pont emplea la formula $\mathrm{Du}$ Pont modificada ${ }^{10}$. Esta fórmula relaciona el rendimiento sobre los activos totales de la empresa (ROA) con su retorno sobre el patrimonio (ROE). Este último se calcula al multiplicar el rendimiento sobre los activos totales por el multiplicador de apalancamiento financiero (MAF), que es la razón entre los activos totales de la empresa y su capital de acciones comunes."

El uso del (MAF) para convertir el (ROA) en (ROE) refleja el impacto del apalancamiento financiero en el retorno de los propietarios.

Figura 2. Representación gráfica de la fórmula Du Pont (Modificado el Margen de Apalancamiento Financiero.

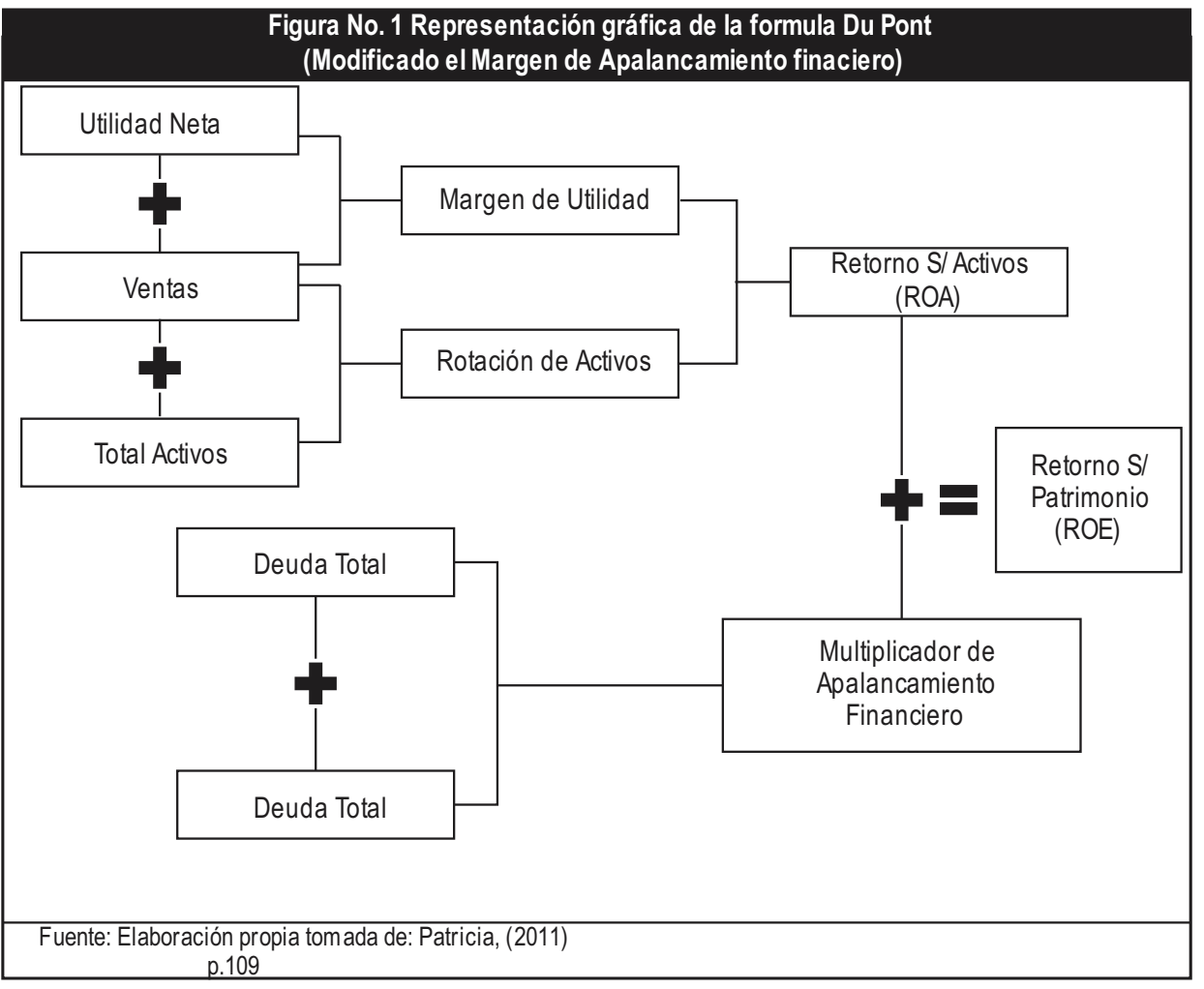

Fuente: Elaboración propia a partir de Mercado (2011)

Como ventaja del sistema Du Pont podemos decir que permite a la empresa dividir el retorno en un componente de utilidad sobre las ventas, un componente de eficiencia del uso de activos y un componente de apalancamiento financiero; es decir, que el Análisis Du Pont nos dice que el ROE se ve afectado por tres cosas:

10 ROE $=$ ROAX MAF 
- $\quad$ Eficiencia operativa, que se mide por el margen de beneficio.

- Eficiencia en el uso de activos, que se mide por el volumen total de activos.

- El apalancamiento financiero, que se mide por el multiplicador de la equidad.

\section{Indices de rentabilidad}

En términos simplificados la rentabilidad se define como la capacidad de producir beneficios (Diccionario Larousse, s.f); con base en esto, se pretende asegurar que una empresa o proyecto sea redituable, maximizando las ganancias y minimizando los costos.

James Van Horne (2002). Nos plantea en una de sus autorías el índice de rentabilidad como la razón costo-beneficio; es decir, la relación entre el valor presente de los flujos futuros de efectivo y el gasto inicial. En otras palabras el objetivo de los indicadores de rentabilidad es establecer y expresar en porcentaje la capacidad de cualquier ente económico para generar ingresos.

Para Companys Pascual (1988) algo es rentable si el valor de sus rendimientos supera al de los recursos utilizados, concretamente, si el valor de los movimientos de fondos positivos supera al de los negativos. Las personas que destinan sus recursos a una empresa, independientemente de su calidad de acreedores o accionistas tienen en mente el objetivo de obtener utilidades sobre la inversión que están efectuando; los acreedores esperan recibir sus utilidades en forma de intereses y los accionistas esperan recibirlas en forma de dividendos y/o ganancias de capital (Ochoa, 1988).

Contemplando que un indicador hace más fácil la interpretación de la rentabilidad; esta situación nos permite analizar de diferentes formas los estados financieros.

Para evaluar la situación de una empresa se requiere indiscutiblemente de ciertos parámetros o criterios. Estos factores alternos son utilizados usualmente como razones o índices.

El análisis e interpretación de las razones en conjunto permite al gerente financiero poseer el dominio y conocimiento integral de la entidad. Para esto se ejecutan dos variables dentro del análisis, el método por tendencias donde se manejan proyecciones y registros anteriores, con el afán de determinar una situación óptima y el método comparativo donde se cotejan resultados entre dos empresas similares. (Van Horne, 1997)

Las razones financieras de diversas industrias son publicadas por Robert Morris Associates, Dun \& Bradstreet, Prentice Hall (Almanac of Business and Industrial Financial Ratios) ${ }^{11}$

Una razón financiera es la división de una cantidad entre otra. En el análisis financiero se comparan las diferentes cuentas de los estados financieros entre sí. Las razones financieras forman parte del análisis en materia financiera más conocidas

11 Podremos observar la publicación de promedios industriales con base a estados financieros, calculando anualmente entre 16 razones financieras para más de 150 líneas de negocios. Almanac of Business and Industrial Financial Ratios(Englewood Cliffs, NJ: Prentice Hall,1994) 
y ampliamente utilizadas, en la mayor parte de los casos proporcionan las claves y los síntomas de las condiciones subyacentes que se analizan y conducen hacia áreas que requieren investigación y examen más profundos. (Morales y Morales 2007)

\section{Cuatro estados financieros básicos}

En relación con las Normas de Información Financiera NIF (2014) se establece que para presentar adecuadamente la realidad económica y financiera de una entidad hay que integrar cuatro estados financieros que la profesión contable considera básicos. ${ }^{12}$

Los cuatro estados financieros clave que se requieren son:

- Estado de resultados: Proporciona un resumen financiero de los resultados operativos de la empresa durante un periodo específico.

- Estado de situación financiera: Refleja la relación que guardan los bienes y derechos de una entidad económica respecto a sus obligaciones con terceros y de la inversión de los socios.

- Flujo de Efectivo: Muestra las fuentes u orígenes de recursos que obtiene la organización lucrativa, así como las aplicaciones o inversiones que efectúa de dichos recursos durante un periodo específico.

- Estado de Variaciones en el Capital Contable, Estado de Variaciones en el Patrimonio, Estado de Utilidades Retenidas o Estado de Otros Cambios en el Patrimonio o Capital: Muestra la eficiencia con la que la inversión de los socios genera utilidades y rendimientos; además, refleja la estructura del capital contable.

Indirectamente la interpretación y manejo adecuado de los estados financieros permite llegar a desarrollar de una manera eficiente la formula Du Pont.

\section{Clasificación de las razones financieras}

La implementación de medidas regulatorias o correctivas dentro de las entidades ha llegado a solventar equitativamente la carencia de rentabilidad. La forma en que nos acercamos o en que mantenemos cierta distancia con el vecino del norte (Estados Unidos) permea y prevalece no sólo el conjunto de las relaciones que sostenemos con el resto del mundo, sino también la vida pública nacional (Navarrete, 2006).

La imitación en forma general muestra el prototipo o modelo económico que pretenden seguir las empresas locales; en otras palabras, la adaptación de las razones financieras deja claro que existe una discrepancia general para el uso de estas que se origina a raíz de las distintas variables que pueden surgir en la economía nacional mexicana.

Las razones financieras para su mejor manejo se han clasificado en cinco tipos: Liquidez, Deuda, Rentabilidad, Cobertura y de Mercado. Justificando la existencia de cada una de las razones y su interacción con las demás cabe resaltar que ninguna razón nos proporciona información suficiente para juzgar las condiciones y desempeño financieros de la empresa

\footnotetext{
12 NIF A-1, (2014)
} 
A su vez las razones de liquidez, actividad y deuda miden principalmente el riesgo. Las razones de rentabilidad miden el retorno y las razones de mercado determinan mutuamente el riesgo y retorno.

\section{Uso e implementación de las razones financieras}

Debemos de mantener en mente que el uso de una sola razón financiera no proporciona la información necesaria para evaluar el rendimiento general de la empresa; de esta forma la única manera de poder determinar o implementar medidas correctivas sería la aplicación general de un conjunto de razones. Otro punto a considerar muy destacable, es que por lo regular se requiere un análisis adicional para cercar las causas del problema; pues el continuo seguimiento a estas razones solo nos muestra los síntomas de un problema.

Como una constante para la determinación de la situación de la entidad podemos utilizar estados financieros del mismo periodo, de periodos anteriores o bien de otra empresa que pertenezca al mismo giro y sector. Pues solo de esta manera podríamos determinar algún bienestar impactante.

\section{Índices de rentabilidad}

a) Margen de utilidad. Indiscutiblemente para determinar el margen de utilidad se debe desarrollarla siguiente formula:

\section{Margen de Utilidad}

Esta fórmula nos permite medir la eficiencia que tiene la empresa para generar utilidades a partir de cada peso vendido. Es importante subrayar que el hecho de vender más, no significa que seamos más rentables, ya que influyen directamente los costos y gastos que se originan por la venta.

\section{b) Retorno sobre activos (inversión)}

Retorno s/activos $=$

$\mathrm{O}$ bien $=$

El primer término de la multiplicación se obtiene directamente del margen de utilidad previamente descrito en el inciso a, multiplicado por la rotación de ventas sobre el total de activos, esta rotación de ventas nos permite medir la capacidad que tienen los activos para generar ventas. En ocasiones se hacen inversiones muy fuertes en activos fijos que no se están utilizando de acuerdo con su capacidad operativa y no están produciendo ventas. Es importante cuidar las inversiones necesarias en activos de la empresa para generar ventas.

\section{c) Retorno sobre patrimonio}

Retorno s/patrimonio $=$

Permite medir la capacidad que tiene la utilidad del ejercicio respecto de la inversión hecha por los accionistas, es decir por cada peso aportado o ganado por la empresa le corresponde a los accionistas vía utilidad del ejercicio.

Razones de liquidez 
La liquidez de una empresa inicialmente se mide con base en su capacidad de respuesta ante sus obligaciones a corto plazo. Al hablar de liquidez tenemos que hacer mención de solvencia general de la empresa; en otras palabras es la facilidad con la que la empresa puede pagar sus deudas.

a) Razón corriente o también conocida como la liquidez corriente: Una de las razones financieras citadas con mayor frecuencia que permite medir la capacidad de la empresa para cumplir con sus obligaciones a corto plazo. Para obtener la liquidez corriente, es necesario aplicar la siguiente fórmula:

Liquidez corriente $=$

Teóricamente el punto para la dictaminación debe de ser 2.0 o 1.0.

b) La prueba de ácido. Es muy similar a la anterior con la diferencia de que a esta no se le contemplan los inventarios, unos de los activos corrientes menos líquido.

Prueba de ácido $=$

Como con la razón Corriente se recomienda el resultado de 1.0 o superior.

Cabe hacer mención que hoy en día uno de los activos de mayor conversión al efectivo lo representan los inventarios, ya que estos se pueden ofrecer a los proveedores o a la competencia, a un precio menor para obtener dinero de manera más rápida. En cambio el uso del factoraje financiero en sus diferentes modalidades o el descuento comercial, llega a ser un trámite algo complicado y tardado. (Tradicionalmente dentro de la convertibilidad de efectivo más rápida se tiene considerado el empleo de las cuentas por cobrar)

\section{Razones de endeudamiento}

Indica la cantidad de dinero de otras personas que se usa para generar utilidades. Un Gerente Financiero se interesa más en las deudas a largo plazo porque estas comprometen a la empresa con un flujo de pagos constantes con los riesgos inherentes. En teoría mientras mayor sea la deuda adquirida mayor es el riesgo de no cumplir. ${ }^{13}$ Ley General de Sociedades Mercantiles (2014)

a) Índice de endeudamiento. Mide la proporción de los activos que se financian por los acreedores.

Índice de Endeudamiento $=$

Se determina en términos porcentuales; mientras mayor sea el resultado más alto es el grado de endeudamiento.

\section{Índices de rotación de activos}

a) Rotación de inventarios. Mide el número de veces que se usan los inventarios de una empresa. Para obtener el índice de rotación debemos aplicar la siguiente fórmula:

Rotación de Inventarios $=$

13 Art. 229 de la Ley General de Sociedades Mercantiles las sociedades se disuelven: ...V.- Por la pérdida de las dos terceras partes del capital social. 
Y para obtener la rotación en días se debe dividir los 365 días entre el índice de rotación de inventarios como sigue:

Rotación en días $=$

Este resultado es interpretado como el número promedio de días que se requieren para vender el inventario.

b) Rotación de cuentas por cobrar o recaudo. El periodo promedio de cobro es útil para evaluar políticas de cobro por parte del departamento de crédito y cobranza.

Promedio de cobro $=$

Promedio de cuentas por cobrar $=$

Promedio en días de recuperación de la cartera o de las cuentas por cobrar es igual a:

Rotación en días $=$

Con base en el resultado podemos determinar las medidas correctivas o modificación en políticas de cobro.

Los posibles prestamistas y proveedores se interesan más en los resultados que arroja esta misma, ya que muestra información sobre los patrones de cobro de los clientes.

c) Rotación de activos fijos. Nos muestra la eficiencia con la cual la empresa utiliza sus activos para generar el flujo de efectivo.

Rotación de activos fijos $=$

El resultado es el número de veces que la empresa cambia sus activos fijos en el año.

d) Rotación de activos totales. Nos muestra la capacidad que tienen los activos totales de la empresa para generar ventas.

Rotación de activos Totales $=$

\section{Razones de cobertura}

a) Cobertura de intereses. Mide la capacidad de la empresa para efectuar pagos fijos derivados de intereses por préstamos solicitados.

Cobertura de Intereses $=$

b) Cobertura de cargos fijos. Mide la capacidad de la empresa para cumplir con todas sus obligaciones. Mientras más alto el resultado mejor.

Cobertura de Cargos fijos $=$

Análisis de la rentabilidad financiera a través de la fórmula Du Pont

Es importante considerar cada aspecto que interviene en la ecuación de la formula 
Du Pont hasta el mayor detalle posible, con el objeto de determinar los valores que están variando el resultado posible, así como establecer las causas que motivan dicho resultado una vez que se hayan detectado las variaciones. El análisis económico utiliza estimaciones de sucesos futuros para ayudar a quienes toman decisiones. Dado que las estimaciones futuras siempre tienen alguna medida de error, hay imprecisión en las proyecciones económicas. El efecto de la variación puede determinarse mediante el análisis de sensibilidad de las variables. (Tarquin, Blank, 2001)

El objetivo de estudio de las cifras en materia financiera de las organizaciones consiste en la determinación de las causas de hechos del pasado y del presente. La interpretación es la base en la que se fundamentan los planes para el futuro, por otra parte resulta conveniente considerar el juicio, experiencia, pericia, conocimiento del negocio, del mercado en particular y en lo general para que el tomador de decisiones pueda hacer una correcta (o incorrecta) interpretación de las cifras, es decir, se deben considerar aspectos cuantitativos y cualitativos. (Morales y otros, 2011)

Existen 2 métodos de análisis para la interpretación de la información financiera los cuales son:

Figura 3. Métodos de Análisis financieros

\begin{tabular}{|c|c|c|c|}
\hline & Internos & $\begin{array}{l}\text { Verticales } \\
\text { Horizontales }\end{array}$ & $\begin{array}{l}\text { Porcientos Integrales } \\
\text { Razones simples } \\
\text { Razones estándar } \\
\text { Aumentos y disminuciones } \\
\text { Tendencias } \\
\text { Control Presupuestal }\end{array}$ \\
\hline $\begin{array}{c}\text { Métodos de } \\
\text { Análisis }\end{array}$ & Externos & \multicolumn{2}{|c|}{$\begin{array}{l}\text { Ambiente } \\
\text { Dirección } \\
\text { Productos y proceso } \\
\text { Función financiera } \\
\text { Facilidad de producción } \\
\text { Materia Prima } \\
\text { Mano de obra } \\
\text { Mercadotecnia, Estadística, Contabilidad, etc. }\end{array}$} \\
\hline
\end{tabular}

Fuente: Elaboración Propia

Dentro del análisis que se hace de manera interna, corresponde exclusivamente a la empresa el poder cuantificar, clasificar, analizar e interpretar los datos que la misma organización genera al realizar transacciones con otros entes económicos, datos que se agrupan para formar la información general de la empresa y específicamente, la información de carácter financiero.

El método vertical consiste en analizar la información financiera correspondiente a un solo periodo de tiempo, mientras que los métodos horizontales o también llamados dinámicos se utilizan para analizar dos periodos dados de la misma empresa en fechas distintas para efecto de poder hacer comparaciones en un 
ejercicio, fecha o periodo dado con respecto al anterior.

El análisis que se realiza de manera externa, se evalúa el impacto que la empresa hace al exterior, es decir, hacia el medio ambiente y de la misma manera como ese medio ambiente también afecta a la empresa. Conviene destacar el hecho por ejemplo, de ver si la empresa cumple con las reglamentaciones legales impuestas por el gobierno en sus tres ámbitos de competencia (Federal, Estatal, Municipal), ubicación de las instalaciones, si está cumpliendo con la parte de Responsabilidad Social Corporativa, facilidad o dificultad de algún insumo, fácil obtención de mano de obra calificada en el mercado laboral, relaciones con instituciones financieras, clientes o proveedores, etc.

A continuación se conceptualizan algunos métodos de análisis financiero internos y externos, tanto verticales como horizontales.

\section{Verticales Internos:}

Método de porcientos integrales: Se convierten las cantidades contenidas tanto en el Estado de Situación Financiera como en el Estado de Resultados a porcientos, en el caso del Estado de Resultados, las ventas constituyen el 100\% o total de la información y a partir de este renglón se analizan los demás. En el Estado de Situación Financiera el total de activos representa el 100\%.

Razones Simples: Consiste en determinar las diferentes relaciones que existen al comparar las cifras de dos o más conceptos que integran los Estados Financieros de la compañía. Representa en términos porcentuales la relación que guardan dos cifras que se quieren comparar entre sí.

\section{Verticales Externos:}

Razones Estándar: Es una relación promedio de los valores de varias empresas con respecto de una razón financiera específica, por ejemplo el margen de utilidad que tienen las empresas que pertenecen al mismo sector, rama y giro de actividades con respecto a esa misma razón que tiene la empresa o bien determinar el porcentaje de calidad con respecto a la producción total de este periodo con respecto a los meses anteriores y analizar las variaciones en caso de que existan.

\section{Horizontales Internos:}

Aumentos y Disminuciones: Permite estudiar los cambios ocurridos de un periodo a otro, en la situación financiera de un ente económico. Es bastante útil el empleo de un balance comparativo para determinar las variaciones tanto positivas como negativas.

Tendencias: Permite a través de las cifras históricas previamente analizadas, hacer pequeñas inferencias y con base en éstas llegar a predecir el comportamiento futuro que tendrá la empresa. Es importante considerar dentro de este método los ciclos del producto, bien sea estacional o cíclica y permanente, la calidad de la gerencia respecto al riesgo, entre otros y no solamente basarse en los informes cuantitativos. Un ejemplo de este tipo de método lo constituye la regresión lineal al tratar de inferir el comportamiento de las ventas futuras.

Control Presupuestal: El presupuesto comprende un programa financiero, estimado para las operaciones de un periodo futuro y el control se ejerce cuando se comparan los resultados presupuestados, para guiar a la empresa en sus 
actividades conforme a los planes financieros. Hablar del éxito de un presupuesto, no quiere decir necesariamente que los resultados logrados hayan sido idénticos a los pronosticados, sería suficiente el haber obtenido una mejoría en el grado de eficiencia y seguridad con que se condujo la Empresa. (Del Río, 2009)

\section{Elementos a considerar dentro de la fórmula Du Pont.}

A partir del método deductivo, es posible estudiar las variaciones porcentuales (positivas y negativas) de cada componente que interviene en el Retorno de la Inversión. Para obtener las variaciones de cada rubro en específico, es necesario utilizar la tasa de cambio que se obtiene de la siguiente manera:

Con el cálculo de las razones financieras se obtiene información expresada en términos porcentuales que permiten hacer comparaciones y análisis tomando como referencia las cifras históricas contenidas dentro de los principales Estados Financieros. El resultado obtenido por sí solo representa un índice, pero se requiere tener un conocimiento, habilidades, pericia para interpretarlos y darle la significación correcta y así obtener en la medida de lo posible una buena interpretación.

Una herramienta importante para profundizar en el análisis de cada componente de la formula Du Pont para el lograr el mayor detalle posible, consiste en detectar los renglones en los que se generan las variaciones (análisis de sensibilidad de las variables) lo constituye la matriz de Fortalezas - Debilidades como factores internos y como factores externos las Oportunidades - Amenazas (FODA o DAFO).

El método 5 W'S y $1 \mathrm{H}$

El método 5 W's y $1 \mathrm{H}$ es una herramienta de análisis que apoya la identificación de los factores y condiciones que provocan problemas en los procesos de trabajo o la vida cotidiana. Las $5 \mathrm{~W}$ 's y $1 \mathrm{H}$ provienen de la primera letra de las siguientes preguntas en inglés: Who (Quién), What (Qué), Where (Dónde), When (Cuándo), Why (Por qué) y How (Cómo). ${ }^{14}$

14 Secretaria de la Función Pública, (2008) "Herramientas para el análisis y Mejora de procesos" manual dy $1 \mathrm{Hel}$ programa especial de mejora en la administración pública federal 2008-2012. 
El siguiente cuadro plantea la mejor forma de tomar en cuenta cada una de las variables posibles:

Tabla 1. Guía de Información de las 5W“s y $1 \mathrm{H}$

\begin{tabular}{|c|c|c|c|}
\hline Who & Quién & Participa en el problema & $\begin{array}{l}\text { Personas de la organización, } \\
\text { proveedores clientes o visitantes } \\
\text { que están presentes o forman } \\
\text { parte de la situación a resolver }\end{array}$ \\
\hline What & Qué & Es el problema & $\begin{array}{l}\text { Delimitar las características } \\
\text { del problema } \\
\text { a) Materiales } \\
\text { b) Humanas } \\
\text { c) Logísticas } \\
\text { d) Tecnológicas } \\
\text { e) Financieras } \\
\text { f) Relación e impacto entre } \\
\text { características } \\
\text { g) Efectos ocasionados por } \\
\text { el problema }\end{array}$ \\
\hline When & Cuando & Ocurre el problema & $\begin{array}{l}\text { Identificar el momento, } \\
\text { horario o epoca del año en que } \\
\text { ocurre el problema, asi como } \\
\text { el punto en el diagrama de flujo } \\
\text { de actividades en que sucede. } \\
\text { Es importante identificar en el } \\
\text { diagrama de flujo en el punto en } \\
\text { que ocurre el problema. }\end{array}$ \\
\hline Why & Donde & Ocurre el problema & $\begin{array}{l}\text { Define "la zona de conflicto" } \\
\text { ya sea por su ubicación física } \\
\text { en las instalaciones de la } \\
\text { organización, o el proceso de } \\
\text { trabajo del que se trate. }\end{array}$ \\
\hline Where & Por qué & $\begin{array}{l}\text { Ocurre el problema, } \\
\text { cual es la ruta }\end{array}$ & 1. ¿Porqué ocurre el problema? \\
\hline How & Cómo & Ocurre el problema & $\begin{array}{l}\text { Secuencia de sucesos que } \\
\text { desencadenan o forman el } \\
\text { problema }\end{array}$ \\
\hline
\end{tabular}

Fuente: Elaboración Propia

Matriz fortalezas, oportunidades, debilidades y amenazas (FODA) y su relación con la formula Du Pont

El análisis $\mathrm{FODA}^{15}$ es una herramienta de carácter gerencial válida para las organizaciones privadas y públicas, la cual facilita la evaluación situacional de la organización y determina los factores que influyen y exigen desde el exterior hacia la entidad. (Zambrano, 2006)

Fortalezas: Son aquellas características propias de la empresa que le facilitan o favorecen en el logro de los objetivos.

Oportunidades: Aquellas ocasiones que se presentan en el entorno de la empresa y que podrían favorecer el logro de los objetivos.

15 A su vez, estos conceptos son la traducción de cuatro iniciales (Strengths, Weaknesses, Opportunities, Threats). Conocido también como análisis "SWOT". 
Debilidades: Aquellas partes que obstaculizan de forma interna el logro de objetivos.

Amenazas: Son factores externos que pueden afectar de manera negativa las posibilidades de logro de los objetivos.

La matriz FODA es una herramienta de análisis que puede ser aplicada a cualquier situación, individuo, producto, empresa, etc., que esté actuando como objeto de estudio en un momento determinado del tiempo.

\section{Análisis FODA y su relación con la formula Du Pont}

De acuerdo con Borello (1994), existen dos determinantes dentro del análisis para su aplicación; Internas (Fortalezas y Debilidades) y externas (Oportunidades y Amenazas).

Al definir las partes internas de la organización, se debe actuar directamente sobre ellas. En cambio las oportunidades y las amenazas son externas, y solo se puede tener injerencia sobre ellas modificando los aspectos internos.

El punto clave del análisis FODA es la implementación de una crítica constructiva; es decir una retroalimentación. Inicialmente tenemos que determinar las variantes de los cuatro rubros y a partir de esto empezar a desarrollar las medidas correctivas que en su caso nos pueden ayudar a mejorar la rentabilidad aplicando la formula Du Pont.

Tabla 2. "Aspectos a considerar dentro la Matriz FODA"

\begin{tabular}{|c|c|}
\hline Fortalezas & Debilidades \\
Buen ambiente laboral & Maquinaria obsoleta \\
Conocimiento del mercado & Falta de capacitación \\
Buen flujo de Efectivo & Mala situación financiera \\
Buena calidad del producto final & Capital de trabajo mal utilizado \\
\hline Oportunidades & Amenazas \\
Competencia débil & Conflictos gremiales (Huelgas, Sindicatos) \\
Necesidad del producto (Demanda) & Cambios en la legislación \\
Inexistencia de competencia & Aumento de precio de insumos \\
& Tendencias desfavorables en el mercado \\
\hline
\end{tabular}

Fuente: Elaboración propia. 


\section{Matriz FODA y la fórmula Du Pont}

La toma de decisiones es un proceso cotidiano mediante el cual se realiza una elección entre diferentes alternativas tanto como muy complejas como multidisciplinarias entre estas se incluyen las de nivel laboral, familiar, sentimental, empresarial, etc., es decir, en todo momento se deben toman decisiones.

Para realizar una acertada toma de decisión sobre un tema en particular, es necesario conocer, comprender y analizar el problema, para así poder darle solución.

Por lo anterior, y antes de tomar cualquier decisión, las empresas deberían analizar la situación teniendo en cuenta la realidad particular de lo que se está analizando, las posibles alternativas a elegir, el costo de oportunidad de elegir cada una de las alternativas posibles, y las consecuencias futuras de cada elección.

Lo significativo y preocupante, es que existe una gran cantidad de empresas que enfrentan sus problemas tomando decisiones de forma automática e irracional (no estratégica), y no toman en cuenta que el resultado de una mala o buena elección puede tener consecuencias en el éxito o fracaso de la misma.

La importancia de confeccionar y trabajar con una matriz de análisis FODA reside en que este proceso nos permite buscar y analizar, de forma proactiva y sistemática, todas las variables que intervienen en el negocio con el fin de tener más y mejor información al momento de tomar decisiones. Los elementos que intervienen en el análisis FODA tienen una repercusión directa en la formula Du Pont, ya que los cambios en las variables o elementos que intervienen en la misma pueden tener su origen en las siguientes consideraciones: 
Tabla 3. Elementos que intervienen en las variaciones del Retorno sobre el Patrimonio.

\begin{tabular}{|l|l|}
\hline$\nabla$ & Tabla 1. "El decrementos que intervienen en la variaciones del Retorno sobre el Patrimonio" \\
\hline$\nabla$ & 1.1 La disminución del Margen de Utilidad de Operación que se origina por: \\
\hline & 1.1.1. Aumento de los costos y gastos dentro del Estado de Resultados \\
\hline & 1.1.2. Disminución de las Ventas o Ingresos \\
\hline$\nabla$ & 1.2 La Disminución de la Rotación del Activo Total se debe a: \\
\hline & 1.2.1. Aumento de los Activos Totales \\
\hline$\triangle$ & 1.2.1. Disminución de las ventas o ingresos \\
\hline$\triangle$ & 2. El aumento del Rendimiento sobre los Activos Totales (ROA) se debe a: \\
\hline & 2.1.1.1 Disminución de los costos y gastos dentro del Estado de Resultados. \\
\hline & 2.1.2. Aumento de las Ventas o Ingresos \\
\hline$\triangle$ & 2.2. El aumento de la Rotación del Activo Total se debe a: \\
\hline & 2.2.1. Disminución de los Acitvos Totales \\
\hline & 2.2.1. Aumento de las ventas o ingresos \\
\hline$\nabla$ & 3.El decremento del Mutiplicador de Apalancamiento financiero propiciado por \\
\hline & 3.1.1. La disminución del activo total \\
\hline & 3.1.2. El aumento del capital contable \\
\hline$\triangle$ & 4.El incremento del Multiplicador de Apalancamiento financiero propiciado por: \\
\hline & 4.1.1 El aumento del activo total \\
\hline & 4.1.1. La disminución del capital contable \\
\hline Fuente: Elaboración Propia \\
\hline
\end{tabular}

Fuente: Elaboración Propia

\section{Metodología de la fórmula Du Pont en cifras}

El análisis económico-financiero puede realizarse a partir del estado de situación financiera y del estado de resultados. Enseguida se mostrarán los 2 estados financieros de manera comparativa de la misma empresa, para enseguida hacer el análisis de la formula Du Pont y hacer las interpretaciones pertinentes. 


\begin{tabular}{|c|c|c|c|c|}
\hline $\begin{array}{l}\text { EMPRESA COMERCIAL, S.A. DE C.V. } \\
\text { ESTADOS DE POSICIÓN FINANCIERA TERI }\end{array}$ & OS EL...... & & & \\
\hline $\begin{array}{l}\text { CONCEPTO } \\
\text { ACTIVO CIRCULANTE }\end{array}$ & $31-\operatorname{dic} 212$ & $31-d$ ic 213 & a riación & \\
\hline BANCOS & 2,850 & 3,000 & $5.26 \%$ & 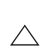 \\
\hline INVERSIONES EN VALORES & 1,250 & 1,100 & $-12.00 \%$ & $\nabla$ \\
\hline CUENTAS POR COBRAR & 3,550 & 3,650 & $2.82 \%$ & $\Delta$ \\
\hline INVENTARIOS & 1,950 & 2,500 & $28.21 \%$ & \\
\hline PAGOS ANTICIPADOS & 450 & 448 & $-0.44 \%$ & \\
\hline SUMA CIRCULANTE & 10,050 & 10,698 & $6.45 \%$ & $\wedge$ \\
\hline PROPIEDADES PLANTA Y EQUIPO - NETO- & 10,500 & 10,150 & $-3.33 \%$ & $\nabla$ \\
\hline TOTAL ACTIVOS & 20,550 & 20,848 & $1.45 \%$ & \\
\hline PASIVO A CORTO PLAZO & & & & \\
\hline $\begin{array}{l}\text { PROVEEDORES } \\
\text { ACREEDORES }\end{array}$ & $\begin{array}{l}1,500 \\
4,500\end{array}$ & $\begin{array}{l}1,230 \\
4,600\end{array}$ & $\begin{array}{r}-18.00 \% \\
2.22 \%\end{array}$ & \\
\hline IMPUESTOS POR PAGAR & 450 & 380 & $-15.56 \%$ & \\
\hline SUMA PASIVO A CORTO PLAZO & 6,450 & 6,210 & $-3.72 \%$ & \\
\hline PASIVO A LARGO PLAZO & 6,500 & 6,000 & $-7.69 \%$ & \\
\hline TOTAL PASIVO & 12,950 & 12,210 & $-5.71 \%$ & $\nabla$ \\
\hline CAPITAL CONTABLE & & & & \\
\hline$\overline{\text { CAPITAL SOCIAL }}$ & 2,500 & 2,500 & $0.00 \%$ & \\
\hline UTILIDADES ACUMULADAS & 3,500 & 5,100 & $45.71 \%$ & \\
\hline UTILIDAD DEL EJERCICIO & 1,600 & 1,038 & $-35.14 \%$ & \\
\hline TOTAL CAPITAL CONTABLE & 7,600 & 8,638 & $13.66 \%$ & \\
\hline SUMA PASICO + CAPITAL & 20,550 & 20,848 & $1.45 \%$ & \\
\hline
\end{tabular}

EMPRESA COMERCIAL, S.A. DE C.V.

ESTADOS DE RESULTADOS DEL 1 DE ENERO AL 31 DE DICIEMBRE DE CONCEPTO 31 -dic212 31 -dic213 Variación

VENTAS

COSTO DE VENTAS

UTILIDAD BRUTA

\begin{tabular}{rrr|r}
14,500 & 15,140 & $4.41 \%$ & $\triangle$ \\
10,150 & 11,052 & $8.89 \%$ & $\triangle$ \\
\cline { 1 - 2 } & $\triangle$
\end{tabular}

GASTOS DE VENTA GASTO DE ADMINISTRACIÓN UTILIDAD DE OPERACIÓN

\begin{tabular}{rrr|r}
1,200 & 1,350 & $12.50 \%$ & $\triangle$ \\
\cline { 1 - 2 } 1,100 & 1,350 & $22.73 \%$ & $\triangle$ \\
\cline { 1 - 2 } \cline { 1 - 2 } 2,050 & 1,388 & $-32.30 \%$ & $\triangle$
\end{tabular}

RESULTADO INTEGRAL DE FINANCIAMIENTO INTERESES PAGADOS

$450 \quad 350-22.22 \% \mid \triangle$

UTILIDAD NETA ANTES DE IMPUESTOS

$1,600 \quad 1,038 \quad-35.14 \%$


Tabla 4. Aplicación de la formula Du Pont (ROE)

\begin{tabular}{|l|r|r|r|r|}
\hline \multicolumn{1}{|c|}{ Razón } & 31 DIC 12 & 31 DIC 13 & \multicolumn{2}{c|}{ Variación } \\
\hline Rentabilidad (ROE) & $21.03 \%$ & $11.99 \%$ & $-42.98 \%$ & \\
\hline Integrada por: & & & & \\
\hline $\begin{array}{l}\text { Rendimiento Sobre } \\
\text { Activos Totales (ROA) }\end{array}$ & $7.78 \%$ & & $-36.11 \%$ & \\
\hline $\begin{array}{l}\text { Multiplicador de } \\
\text { Apalancamiento } \\
\text { Financiero (MAF) }\end{array}$ & $2.70 \%$ & & & $-10.74 \%$ \\
\hline
\end{tabular}

Fuente: Elaboración propia

\section{Análisis Financiero}

Se registra una disminución de 42.98 puntos porcentuales en el Retorno sobre el Patrimonio o inversión (ROE) al pasar de $21.03 \%$ al $11.99 \%$. Esto obedece principalmente a la disminución del rendimiento sobre activos totales (ROA) $36.11 \%$ y a la disminución en el multiplicador de apalancamiento financiero MAF -10.74\%).

Tabla 5. Análisis del Rendimiento sobre Activos ${ }^{16}$

\begin{tabular}{|l|r|r|r|r|}
\hline \multicolumn{1}{|c|}{ Razón } & \multicolumn{1}{c|}{31 DIC 12 } & 31 DIC 13 & \multicolumn{2}{c|}{ Variación } \\
\hline $\begin{array}{l}\text { Rendimiento sobre } \\
\text { activos (ROA) }\end{array}$ & $7.78 \%$ & $4.97 \%$ & $-36.11 \%$ & \\
\hline Compuesto por: & $11.03 \%$ & $6.85 \%$ & $-37.89 \%$ & \\
\hline $\begin{array}{l}\text { Margen de utilidad de } \\
\text { operación }\end{array}$ & $70.55 \%$ & $72.62 \%$ & $2.93 \%$ & \\
\hline Rotación del Activo Total & & & & \\
\hline
\end{tabular}

Fuente: Elaboración propia

\section{Análisis Financiero}

La disminución del 36.11\% en el Rendimiento sobre Activos obedece principalmente a la disminución en la utilidad del ejercicio en un 37.89\% y paradójicamente existe un incremento en la rotación del activo total en un $2.93 \%$. Aunque existe un incremento en la rotación del activo, la disminución en el margen de operación tiene mayor peso, por lo que incide directamente en el resultado negativo, todo ello obedece a la disminución en la utilidad del ejercicio, ya que incluso existe un aumento en las ventas, sin embargo también aumentaron los costos y gastos.

El Rendimiento Sobre Activos evalúa la eficiencia en la administración de la empresa para lograr el nivel de ventas con menos costos y gastos así como la utilidad obtenida, los activos de la empresa en inventarios, activos fijos, y del uso del efectivo. En este 
punto se identifican elementos fuertes y débiles para alcanzar el ROA.

Tabla 6. Análisis del Margen de Utilidad de Operación

\begin{tabular}{|l|r|r|r|r|}
\hline \multicolumn{1}{|c|}{ Razón } & 31 DIC 12 & 31 DIC 13 & \multicolumn{2}{c|}{ Variación } \\
\hline $\begin{array}{l}\text { Margen de utilidad de } \\
\text { operación }\end{array}$ & $11.03 \%$ & $6.85 \%$ & $-37.89 \%$ & \\
\hline Compuesto por: & & & & \\
\hline Utilidad Neta & 1,600 & 1,038 & $-35.12 \%$ & \\
\hline Ventas Totales & 14,500 & 15,140 & $4.41 \%$ & \\
\hline
\end{tabular}

Fuente: Elaboración propia

\section{Análisis Financiero}

El Margen de Utilidad de Operación mide el porcentaje de ganancia (utilidad) por cada peso vendido una vez que se descontaron los costos y gastos. Esta disminución obedece principalmente a la disminución en la utilidad en un $35.12 \%$, no obstante que las ventas aumentaron un $4.41 \%$, también aumentaron los costos y gastos en un importe mayor por lo que no estamos siendo muy eficientes en la manera de controlar y vigilar dichos insumos, como veremos enseguida en la siguiente tabla.

Tabla 7. Análisis de los costos y gastos con respecto de las ventas

\begin{tabular}{|c|c|c|c|}
\hline Razón & 31 DIC 12 & 31 DIC 13 & Variac \\
\hline Ventas & 14,500 & 15,140 & $4.41 \%$ \\
\hline Costo de Ventas & 10,150 & 11,052 & $8.88 \%$ \\
\hline Gastos de Venta & 1,200 & 1,350 & $12.50 \%$ \\
\hline $\begin{array}{l}\text { Gastos de Admón. } \\
\text { ADMINISTRACION }\end{array}$ & 1,100 & 1,350 & $22.72 \%$ \\
\hline Intereses pagados & 450 & 350 & $-22.22 \%$ \\
\hline Utilidad Neta & 1,600 & 1,038 & $-35.12 \%$ \\
\hline
\end{tabular}

Fuente: Elaboración propia 


\section{Análisis Financiero}

Es importante tener el control sobre los insumos como son los inventarios que se venden y formar parte del costo de ventas, los gastos de venta y administración sin descuidar las ventas, por otro lado, se deben vigilar los presupuestos de ventas para efecto de comparar las cifras presupuestadas al inicio de cada periodo contra los resultados obtenidos en la realidad, para determinar y analizar las diferencias correspondientes.

Tabla 8. Análisis de la Rotación del Activo Total

\begin{tabular}{|l|r|r|r|r|}
\hline \multicolumn{1}{|c|}{ Razón } & 31 DIC 12 & 31 DIC 13 & \multicolumn{2}{c|}{ Variación } \\
\hline Rotación del Activo Total & $70.55 \%$ & $72.62 \%$ & $2.93 \%$ & \\
\hline Compuesto por: & 14,500 & 15,140 & $4.41 \%$ & \\
\hline Ventas Totales & 20,550 & 20,848 & $1.45 \%$ & \\
\hline Activos Totales & & & & \\
\hline
\end{tabular}

Fuente: Elaboración Propia

Dentro de los activos totales están incluidos tanto los activos circulantes como las propiedades, planta y equipo que posee la empresa.

Tabla 9 Integración de los Activos Totales

\begin{tabular}{|c|c|c|c|c|}
\hline Razón & 31 DIC 12 & 31 DIC 13 & \multicolumn{2}{|c|}{ Variación } \\
\hline Circulante & 10,050 & 10,698 & $6.44 \%$ & \\
\hline $\begin{array}{c}\text { Propiedades, Planta y } \\
\text { Equipo }\end{array}$ & & & & \\
\hline Activo Total & 10,500 & 10,150 & $-3.33 \%$ & \\
\hline
\end{tabular}

Fuente: Elaboración propia

Tabla 10. Análisis de la Variación en el Multiplicador de Apalancamiento Financiero (MAF) ${ }^{17}$

\begin{tabular}{|c|c|c|c|}
\hline Razón & 31 DIC 12 & 31 DIC 13 & Variación \\
\hline $\begin{array}{c}\text { Multiplicador de } \\
\text { Apalancamiento } \\
\text { Financiero }\end{array}$ & $270.39 \%$ & $241.35 \%$ & $-10.74 \%$ \\
\hline
\end{tabular}

Fuente: Elaboración Propia

\section{Análisis Financiero}

La rotación del activo total aumento en un 6.44\% derivado del aumento en las ventas

17

110

INQUIETUD EMPRESARIAL 
(4.41\%) y de los activos totales que posee la empresa (1.45\%), es importante cuidar esta razón financiera, ya que un aumento en los activos se debe traducir en un aumento en las ventas, de lo contrario estaríamos comprando activos fijos ociosos u ocupándolos en un nivel inferior a su productividad. Las inversiones en activos de larga duración muchas veces son adquiridas con financiamiento a largo plazo, modificando el Multiplicador de Apalancamiento Financiero (MAF) como se verá a continuación.

Se está empleando de manera propia el endeudamiento ya que incluso los pasivos representaron una disminución del $7.69 \%$ del 2013 con respecto al año anterior, lo cual revela que los recursos de terceros como son proveedores, bancos, acreedores se utilizan para impulsar a la empresa y generar utilidades.

\section{CONCLUSIONES}

La fórmula Du Pont plantea una serie de razones financieras muy importantes para medir la eficiencia de la empresa en áreas como son las ventas, al permitir mediante los pronósticos financieros vigilar y controlar que las mismas se vayan alcanzando conforme a los planes que se trazaron al inicio del año. Por otro lado permite evaluar con qué grado de control o eficiencia se están aplicando los costos y gastos para generar las ventas, ya que el hecho de vender mucho, no significa que se es más eficiente. Todos estos factores contribuyen de manera directa en la utilidad de la empresa.

También nos permite vigilar el grado en que los activos de la empresa se están empleando en la generación de los ingresos, ya que normalmente las inversiones hechas en los inventarios, equipo de cómputo, maquinaria, equipo de transporte, etc., se utilizan para generar ventas, por lo que es importante no tener inversiones ociosas o con activos que se estén utilizando con una capacidad inferior a la que los mismos puedan ofrecer.

Es importante controlar las inversiones hechas en activos de larga duración, ya que en la mayoría de las veces se incurre en pasivos para financiar estas operaciones, como son: créditos a proveedores, créditos bancarios, arrendamientos, lease back, leasing, etc., por lo que se genera una carga financiera impactando directamente a la utilidad del ejercicio y por ende al Margen de Apalancamiento Financiero.

A través del Estado de Resultados y del Estado de Situación Financiera, se obtienen los datos relativos para la elaboración de la Formula Du Pont. Una vez hechas las operaciones para obtener las razones financieras, es importante que se evalúen de manera conjunta con otras herramientas financieras como pueden ser los análisis de sensibilidad, el uso de la matriz FODA.

Cuando se analicen los índices financieros para corregir alguna deficiencia encontrada, se sugiere que intervengan factores cualitativos como pueden ser el grado de conocimiento de la empresa, la pericia del tomador de decisiones, la experiencia, el mercado, cuestiones externas que afecten de manera interna a la organización, etc., para no incurrir en algunas desviaciones y que incidan al error.

Una de las principales metas o funciones que tiene un analista financiero, gerente financiero o persona encargada de los funciones de manejar la rentabilidad de una empresa, es maximar el rendimiento neto sobre las inversiones hechas por los accionistas o en su defecto el minimizar costos y/ gastos, todo ello en aras de 
incrementar las utilidades, base para el pago de dividendos o intereses en caso de que se haya financiado la empresa con pasivos, ya sean a corto o a largo plazo.

De ahí la importancia que revisten algunas funciones o actividades administrativas, ya que necesariamente cualquier decisión o falta de ellas, se traducirán en eficiencias o deficiencias financieras, por lo que es importante que periódicamente se vayan evaluando los resultados obtenidos contra los presupuestos establecidos dentro de la planeación estratégica.

Aunque existe mucha literatura sobre la formula Du Pont, el presente trabajo contribuye a crear esa simbiosis entre el ámbito financiero con algunas herramientas administrativas que permitan hacer una mejor interpretación de los resultados obtenidos al emplear la formula Du Pont. De la misma manera se presenta un caso práctico para su fácil aplicación y con algunas reflexiones que se hacen por cada elemento analizado, y que contribuya con la academia, empresarios y profesionistas como una guía en materia financiera para toma de decisiones.

\section{REFERENCIAS BIBLIOGRÁFICAS}

Block, S. y Hirt, A. (2000). "Fundamentos de Gerencia Financiera". Colombia, McGraw Hill

Borello, A. (1994). "El Plan de Negocios”. Madrid España, Ed. Ediciones Díaz de Santos, $1^{\text {a }}$.Edición

Catanese, L. (1997). "Women's history: a guide to sources at Hagley Museum and Library". USA. Library of congress cataloging in publication data, $1^{\text {st }}$ published.

Chandler, J. y Alfred, D. (2003). "Strategy and Structure: Chapters in the History of the American Industrial Enterprise". Washington, D.C. United States of America, Ed. Beard Books.

Comellas, J. (2004). "Historia Breve del Mundo Contemporáneo”. Madrid, España Ed. RIALP, Cuarta Edición.

Companys, R. (1988). "Planificación Rentabilidad de Proyectos Industriales”. Barcelona España ed. Marcombo Boixareu

Del Río Sánchez, C., Del Río Sánchez, R. y Del Río González, C. (2009). "El Presupuesto". México. Cengage Learning Editores, S.A. de C.V.

Diccionario Larousse en línea. (s.f). Diccionario. Recuperado 30/05/2014 de: http:// www.larousse.com.mx/Home/Diccionarios/rentabilidad

Dupont. (2014). “1918 DuPont, GM \& Cars”. Recuperado 01/06/2014 http://www2. dupont.com/Phoenix_Heritage/en_US/1918_detail.html 
FÓRMULA DU PONT Y SU RENTABILIDAD, VISTA DESDE LA OPTICA ADMINISTRATIVA

Enciclopedia Britanica. (2014). “Great Depression”. Recuerado 02/06/2014 de: http://www.britannica.com/EBchecked/topic/243118/Great-Depression

Gitman, L. (2007). "Principios de Administración Financiera”. México, Pearson Educación.

Instituto Mexicano de Contadores Públicos (IMCP), Consejo Mexicano de Normas de Información Financiera, A.C (CINIF), (2014) "Normas de Información Financiera", México, 9a edición

Ley General de Sociedades Mercantiles (2014) Articulo 229, México, Editorial Porrúa Hermanos.

Mercado, P. (2011). "Temas selectos sobre áreas funcionales de las organizaciones”. México Bonobos Editores, S. de R.L. de C.V. 1ª Edición.

Morales, A., Morales, J. y Alcocer, F. (2011). “Administración Financiera”. México, Grupo Editorial Patria.

Morales, A. y Morales, J. (2007). "La valuación del desempeño de los administradores de empresas”. Revista Emprendedores. No. 106 p. 21.

Navarrete, J. (2006). "La reconstrucción de la Política Exterior De México: Principios, Ámbitos, Acciones”. México, primera edición, ed. Centro de Investigaciones Interdisciplinarias en Ciencias y Humanidades, UNAM

Ochoa, G. (1998). “Administración Financiera I”. México, Ed. Pearson Education Inc., Tercera Edición.

Tarquin, A. y Blank, L. (2001). "Ingeniería Económica”. Colombia, McGraw Hill

Van Horne, J. (2002). "Fundamentos de Administración Financiera". México, Ed. Pearson Education Inc.

Zambrano, A. (2006). "Planificación Estratégica, presupuesto y control de la gestión pública”. Caracas Venezuela ed. Texto C.A. 